\title{
Determinação do volume, do fator de forma e da porcentagem de casca de árvores individuais em uma Floresta Ombrófila Aberta na região noroeste de Mato Grosso
}

\author{
Chirle COLPINI ${ }^{1}$, Danielle Parra TRAVAGIN ${ }^{21}$ Thelma Shirlen SOARES ${ }^{2 *}$,
}

Versides Sebastião Moraes e SILVA ${ }^{3}$

\section{RESUMO}

O objetivo deste estudo foi testar e selecionar modelos que expressam o volume com e sem casca e determinar o fator de forma e a porcentagem de casca para uma área de floresta ombrófila aberta na região noroeste de Mato Grosso. Foi realizada a cubagem rigorosa de 91 árvores para a obtenção do diâmetro, espessura de casca, altura total do fuste e volume sólido. Dez modelos volumétricos foram testados, sendo que para a seleção do melhor modelo foram usadas as estatísticas do coeficiente de determinação ajustado, erro padrão da estimativa, seguida da análise de resíduos e distribuição gráfica dos resíduos. Os modelos selecionados foram validados pela aplicação do teste L\&O. O fator de forma médio obtido foi de 0,7424 e 0,7297 com e sem casca, respectivamente. O volume médio de casca foi de $0,4292 \mathrm{~m}^{3}$ (7,45\% do volume total). O modelo de Schumacher-Hall foi o que melhor se ajustou aos dados de volumes com e sem casca.

PALAVRAS CHAVE: Regressão, Fator de forma, Porcentagem de casca.

\section{Determination of bark percentage and volume of individual trees in an Open Ombrophylous Forest in northwest Mato Grosso}

\section{ABSTRACT}

This study aimed to test and select models, with and without bark volume, and to determine the form factor and bark percentage of an open ombrophylous forest in northwest Mato Grosso. 91 trees were cubed by obtaining diameter, bark percentage, stem height and solid stem volume. A total of 10 models were tested. Statistical procedures, such as adjusted determination coefficient, residual error and distribution of residuals, were tested. The selected models were validated by the $\mathrm{L} \& \mathrm{O}$ test. The average form factor was 0.7424 and 0.7297 , with and without bark, respectively. The average volume of bark was $0.4292 \mathrm{~m}^{3}$ (7.45\% of total volume). The Schumacher-Hall model showed the best performance for volumes with and without bark.

KEYWORDS: Regression, Form factor, Bark percentage.

\footnotetext{
Acadêmica do Programa de Pós-Graduação do Curso de Mestrado em Ciências Florestais e Ambientais, Universidade Federal de Mato Grosso, Faculdade de Engenharia Florestal, CEP 78060-900, Cuiabá-MT

2 Professora do curso de Engenharia Florestal, Universidade Federal dos Vales do Jequitinhonha e Mucuri, Departamento de Engenharia Florestal, CEP 39100-000, Diamantina-MG e-mail: thelma.soares@ufvjm.edu.br *Autor de correspondência

3 Professor do curso de Engenharia Florestal, Universidade Federal de Mato Grosso, Faculdade de Engenharia Florestal, CEP 78060-900, Cuiabá-MT.
} 


\section{INTRODUÇÃO}

A obtenção de estimativas precisas de produtividade em formaçōes vegetais tropicais é um pré-requisito importante no estabelecimento de açōes de manejo (Scolforo, 1997). Porém, para a vegetação amazônica, devido, principalmente, à diversidade de espécies, estudos de tais estimativas ainda são escassos.

Essa escassez é em virtude do tema volumetria já ter sido muito difundido e estudado por vários pesquisadores como Chapman \& Meyer (1949), Bruce \& Schumacher (1950), Spurr (1952), dentre outros, no Brasil os estudos são desenvolvidos desde a década de 1970 por Paula Neto (1977), Siqueira (1977), Higuchi et al. (1979), Machado (1979), dentre outros. Entretanto, mesmo com esta vasta experiência, na Amazônia não existem equaçôes de volume que representem a maioria das espécies arbóreas nos diferentes sítios e regiōes.

No estado de Mato Grosso a situação é ainda mais agravante, uma vez que o Estado é desprovido destes estudos e os poucos modelos volumétricos que foram desenvolvidos não estão disponíveis para o público interessado nos mesmos.

De acordo com Scolforo et al. (1994) a existência de relaçôes quantitativas e modelos matemáticos que sejam consistentes e numericamente compatíveis para a prediçāo do desenvolvimento do povoamento de qualquer idade, além de permitir o monitoramento do desenvolvimento de espécies florestais nativas, é elemento fundamental no manejo florestal sustentável.

O volume constitui uma das informações de maior importância para o conhecimento do potencial disponível em um povoamento florestal, tendo em vista que o volume individual fornece subsídios para a avaliação do estoque de madeira e análise do potencial produtivo das florestas (Thomas et al., 2006).

Outra informação relevante é o percentual de casca, visto que este é um aspecto importante no processo de avaliação e comercialização das toras obtidas em uma floresta. De acordo com Scolforo \& Thiersch (2004), a espessura de casca varia consideravelmente entre espécies, dentro de uma mesma árvore, de local para local e de acordo com a idade, dentre outros.

O objetivo deste estudo foi ajustar e selecionar modelos que expressam o volume com e sem casca, determinar o volume de casca em porcentagem e o fator de forma para a Floresta Ombrófila Aberta na região noroeste de Mato Grosso.

\section{MATERIAL E MÉTODOS}

\section{LOCALIZAÇÃO E CARACTERIZAÇÃO DA ÁREA DE ESTUDO}

Os dados utilizados para este estudo foram provenientes de uma área de floresta nativa do bioma Amazônia, pertencente à empresa ONF Brasil, localizada no município de Cotriguaçú, região noroeste do Estado de Mato Grosso, entre os paralelos $9^{\circ} 47^{\prime}$ e $9^{\circ} 53^{\prime}$ de latitude sul e os meridianos $58^{\circ} 13^{\prime}$ e $58^{\circ} 19^{\prime}$ de longitude oeste de Greenwich, com altitude variando entre 100 e $150 \mathrm{~m}$.

O clima da região é do tipo tropical chuvoso (Am de Köppen) caracterizado por apresentar temperatura média anual de $24^{\circ} \mathrm{C}$. A precipitação média anual é de $2.500 \mathrm{~mm}$, sendo o período chuvoso ocorrente nos meses da primaveraverão, com curto período seco no inverno (Brasil, 1982).

O solo predominante na região é o podzólico vermelho amarelo distrófico e álico com textura argilosa, ocorrendo ainda, em menores proporçôes, os solos litólicos, em locais de relevo mais movimentado e solos hidromórficos.

\section{FONTE DE DADOS}

As árvores que constituíram a amostra, em número de 91, foram cubadas pelo método de Smalian. As circunferências foram tomadas as alturas de $0,20 \mathrm{~m}, 1,30 \mathrm{~m}, 2,0 \mathrm{~m}$ e em intervalos regulares de 2,0m até a base da copa. Nas árvores com presença de sapopemas as medidas foram tomadas a $0,30 \mathrm{~m}$ acima destas, com intervalos regulares de 2,0m. Em cada ponto de medição tomou-se as medidas da espessura da casca.

Empregou-se o processo de seleção aleatória das árvoresamostras em função da distribuição em classes de dap (diâmetro medido a 1,3 m de altura do solo), com amplitude da classe de $10 \mathrm{~cm}$, sendo que cada árvore amostra deveria estar representada na diagonal de todas as classes de diâmetro e altura na floresta. A distribuição de frequiência das árvores empregadas na cubagem é apresentada na Tabela 1.

$\mathrm{Na}$ amostra foram consideradas todas as espécies de interesse comercial e as potenciais, com destino para madeira serrada e laminas. As espécies que foram cubadas encontramse relacionadas na Tabela 2. A identificação taxonômica das espécies foi efetuada mediante consultas a herbários, consultas a especialistas e por meio de literatura especializada. A sinonímia e a grafia dos taxa foram atualizadas mediante consulta ao índice de espécies do Royal Botanic Garden e do banco de dados do Missouri Botanical Garden, disponível na página http://mobot.mobot.org/W3T/Search/vast.html, sendo que o sistema de classificação adotado foi o APG II (2003). O material botânico coletado em estado reprodutivo está depositado no Herbário Central da Universidade Federal de Mato Grosso.

Em cada árvore-amostra foram realizadas determinações de altura do fuste até a base da copa (considerada altura comercial) e cap (circunferência medida a 1,3 m de altura do solo). Foram também medidas as circunferências com e sem casca nas secçôes do tronco estabelecidas na cubagem. 
Tabela 1 - Distribuição de freqüência de árvores-amostra utilizadas para ajuste dos modelos.

\begin{tabular}{|c|c|c|c|c|c|c|c|c|c|c|c|c|c|}
\hline \multirow{2}{*}{$\begin{array}{l}\text { Classe de dap } \\
\quad(\mathrm{cm})\end{array}$} & \multicolumn{12}{|c|}{ Classe de altura (m) } & \multirow{2}{*}{ - Total } \\
\hline & $7-9$ & $9-11$ & $11-13$ & $13-15$ & $15-17$ & $17-19$ & $19-21$ & $21-23$ & $23-25$ & $25-27$ & $27-29$ & $29-31$ & \\
\hline $15-25$ & 3 & 5 & 2 & 4 & & & & & & & & & 14 \\
\hline $25-35$ & 1 & & & 4 & 2 & & & & & & & & 7 \\
\hline $35-45$ & & 1 & 4 & 3 & & 1 & & & & & & & 9 \\
\hline $45-55$ & & & 4 & 4 & 1 & & & 1 & & & & & 10 \\
\hline $55-65$ & & & 1 & & 4 & 2 & & & 1 & & & & 8 \\
\hline $65-75$ & & & & 1 & 3 & & 3 & & & & & 1 & 8 \\
\hline $75-85$ & & & & 2 & 3 & & 1 & 2 & & & & & 8 \\
\hline $85-95$ & & & & 2 & 1 & 3 & 1 & 1 & 2 & 1 & & & 11 \\
\hline $95-105$ & & 1 & 1 & 1 & 1 & 1 & 2 & & 1 & & & & 8 \\
\hline $105-115$ & & & & & 1 & & & 2 & 1 & & & 1 & 5 \\
\hline $115-125$ & & & & & 1 & & & & & & & & 1 \\
\hline $125-135$ & & & & & & 1 & & & & 1 & & & 2 \\
\hline Total & 4 & 7 & 12 & 21 & 17 & 8 & 7 & 6 & 5 & 2 & & 2 & 91 \\
\hline
\end{tabular}

Tabela 2 - Relação das espécies arbóreas utilizadas na cubagem

\begin{tabular}{|c|c|c|c|}
\hline Família & Nome Cientifico & Nome Popular & № de indivíduos \\
\hline \multirow{3}{*}{ Anacardiaceae } & Anacardium giganteum W. Hancock ex Engl. & Cajueiro & 3 \\
\hline & Astronium lecointei Ducke & Muiracatiara & 2 \\
\hline & Spondias dulcis Parkinson & Cajá & 1 \\
\hline Apocynaceae & Aspidosperma album (Vahl) Benoist ex Pichon & Perobaud'água & 1 \\
\hline Bignoniaceae & Tabebuia sp. & Ipê & 1 \\
\hline \multirow{2}{*}{ Burseraceae } & Protium robustum (Swart) Porter & Amesclauaroeira & 12 \\
\hline & Trattinnickia sp. & Morcegueira & 2 \\
\hline Caryocaraceae & Caryocar glabrum (Aubl.) Pers. & Piqui & 3 \\
\hline Cecropiaceae & Cecropia sp. & Embaúba & 1 \\
\hline Celastraceae & Goupia glabra Aubl. & Peroba-cupiúba & 1 \\
\hline Combretaceae & Buchenavia sp. & Mirindiba & 1 \\
\hline \multirow{4}{*}{ Euphorbiaceae } & Hevea sp. & Seringueira & 2 \\
\hline & Sapium aereum Klotzsch ex Müll. Arg. & Leiteiro/Cega-corrente & 3 \\
\hline & Sapium bogotense Huber & Caucho & 2 \\
\hline & Sapium sp. & Caucho-vermelho & 1 \\
\hline \multirow{14}{*}{ Fabaceae } & Alexa grandiflora Ducke & Melancieiro & 2 \\
\hline & Apuleia leiocarpa (Vogel) J.F. Macbr. & Garapeira & 2 \\
\hline & Copaifera sp. & Copaíba & 3 \\
\hline & Dialium guianense (Aubl.) Sandwith & Jutaí-pororoca & 1 \\
\hline & Dinizia excelsa Ducke & Angelim-pedra & 1 \\
\hline & Dinizia sp. & Angelim-vermelho & 1 \\
\hline & Enterolobium sp. & Tamboril & 3 \\
\hline & Erythrina fusca Lour. & Mulungú & 1 \\
\hline & Hymenaea courbaril L. & Jatobá & 1 \\
\hline & Hymenolobium pulcherrimum Ducke & Angelim-amarelo & 1 \\
\hline & Parkia pendula (Willd.) Benth. ex Walp. & Angelim-saia & 2 \\
\hline & Peltogyne sp. & Roxinho & 1 \\
\hline & Plathymenia reticulata Benth. & Guariúba/Amarelinho & 1 \\
\hline & Schizolobium amazonicum Huber ex Ducke & Pinho-cuiabano & 1 \\
\hline \multirow{2}{*}{ Lauraceae } & Mezilaurus itauba (Meisn.) Taub. ex Mez & Itaúba & 2 \\
\hline & Ocotea sp. & Canela & 1 \\
\hline
\end{tabular}




\begin{tabular}{|c|c|c|c|}
\hline Família & Nome Científico & Nome Popular & № de indivíduos \\
\hline \multirow{4}{*}{ Lecythidaceae } & Bertholletia excelsa Bonpl. & Castanheira & 3 \\
\hline & Cariniana sp. & Jequitibá & 2 \\
\hline & Couratari guianensis Aubl. & Tauari & 2 \\
\hline & Eschweilera ovata (Cambess.) Miers & Flor-de-paca & 2 \\
\hline Malpighiaceae & Byrsonima densa (Poir.) DC. & Murici & 1 \\
\hline \multirow{2}{*}{ Meliaceae } & Cedrela odorata L. & Cedro-rosa & 1 \\
\hline & Guarea silvatica C. DC. & Cedro-marinheiro & 4 \\
\hline Polygonaceae & Triplaris surinamensis Cham. & Tachi & 2 \\
\hline Sapindaceae & Sapindus cerasinus Benth. & Pitombeira & 1 \\
\hline Sapotaceae & Planchonella sp. & Goiabão & 1 \\
\hline Simaroubaceae & Simarouba amara Aubl. & Marupá & 2 \\
\hline Sterculiaceae & Sterculia apetala (Jacq.) H. Karst. & Amendoim-bravo & 1 \\
\hline Tiliaceae & Apeiba tibourbou Aubl. & Escova-de-macaco & 1 \\
\hline \multirow{3}{*}{ Vochysiaceae } & Erisma uncinatum Warm. & Cambará-rosa & 1 \\
\hline & Qualea sp. & Catuaba & 1 \\
\hline & Vochysia sp. & Rosinha & 2 \\
\hline Não identificado & & & 4 \\
\hline Total & & & 91 \\
\hline
\end{tabular}

As medidas obtidas foram empregadas como referência padrão para ajuste dos modelos volumétricos, sendo que para cada árvore amostrada foram calculados os volumes com e sem casca e, ainda, determinado o fator de forma normal ou comum.

Com os valores da espessura da casca tomados nos pontos de medição pode-se obter um fator ( $\mathrm{k}$ = constante de casca) que permitiu determinar o volume de casca para cada espécie arbórea encontrada no inventário. A fórmula empregada para obtenção do volume de casca em função do volume com casca é dada por:

\section{$\mathrm{Vc}=\operatorname{Vcc} \times\left(1-\mathrm{K}^{2}\right)$}

em que:

$\mathrm{Vc}=$ volume de casca;

$\mathrm{Vcc}=$ volume com casca;

$\mathrm{K}$ = constante de Meyer que é obtida pela razão da soma do diâmetro sem casca pela soma do diâmetro com casca, sendo K o valor médio por árvore.

Para a determinação da quantidade de casca por espécie foi utilizada a equação preconizada por Spurr (1952), Prodan (1968), Machado \& Figueiredo Filho (2003), Scolforo \& Thiersch (2004):

$\mathrm{V}_{\mathrm{C}} \%=\left(1-\mathrm{K}^{2}\right) \times 100$

em que:
$\mathrm{Vc} \%$ = porcentagem de casca de cada espécie ou grupo de espécies;

$\mathrm{K}$ = conforme definido anteriormente.

Foram comparados modelos clássicos de equações volumétricas para selecionar as melhores equações para estimativas dos volumes com e sem casca em função das variáveis dependentes dap (diâmetro medido a 1,3 m de altura do solo) e altura total. Os modelos testados estão relacionados na Tabela 3 .

Os modelos foram ajustados pelo método dos mínimos quadrados descrito por Draper \& Smith (1998). Cada modelo, individualmente, foi avaliado com as seguintes estatísticas: coeficiente de determinação ajustado $\left(\mathrm{R}^{2}{ }_{\text {ajustado }}\right)$, erro padrão da estimativa $\left(\mathrm{S}_{\mathrm{yx}} \%\right)$ e distribuição gráfica dos resíduos. Para fins de comparação dos modelos logarítmicos com os não logarítmicos foi recalculado o erro padrão de estimativa e o coeficiente de determinação conforme recomendado por Machado et al. (2004).

Após selecionar os modelos que apresentaram melhores resultados, verificou-se a validade das estimativas volumétricas desses modelos em relação ao volume real, aplicando-se o teste L \& O, proposto por Leite \& Oliveira (2002), cujo procedimento resulta da combinação do teste de $\mathrm{F}\left(\mathrm{FH}_{0}\right)$ Graybill, do teste $\mathrm{t}$ para erro médio $(\mathrm{t} \bar{e})$ e da análise de correlação linear $\left(r_{Y_{i} Y_{1}}\right)$ entre os valores observados $\left(Y_{j}\right)$ e os valores estimados $\left(Y_{i}\right)$. O teste compara o valor observado, como variável dependente, e o valor estimado, como variável independente. Assim, para situaçóes em que $\mathrm{F}\left(\mathrm{H}_{0}\right)$ e t $\bar{e}$ 


\begin{tabular}{lll}
\hline \multicolumn{2}{l}{ Tabela 3 - Modelos testados para estimar o volume total de árvores individuais do povoamento* } \\
\hline Modelo & Autor & Modelo \\
\hline 1 & Kopezky - Gehrhardt & $\mathrm{v}=\beta_{0}+\beta_{1} \mathrm{~d}^{2}+\varepsilon_{\mathrm{i}}$ \\
\hline 2 & Hohenald - Krenn & $\mathrm{v}=\beta_{0}+\beta_{1} \mathrm{~d}+\beta_{2} \mathrm{~d}^{2}+\varepsilon_{\mathrm{i}}$ \\
3 & Husch & $\ln (\mathrm{v})=\beta_{0}+\beta_{1} \ln (\mathrm{d})+\varepsilon_{\mathrm{i}}$ \\
4 & Brenac & $\ln (\mathrm{v})=\beta_{0}+\beta_{1} \ln (\mathrm{d})+\beta_{2} \frac{1}{\mathrm{~d}}+\varepsilon_{\mathrm{i}}$ \\
\hline 5 & Spurr & $\mathrm{v}=\beta_{0}+\beta_{1} \mathrm{~d}^{2} \mathrm{~h}+\varepsilon_{\mathrm{i}}$ \\
\hline 6 & Stoate & $\mathrm{v}=\beta_{0}+\beta_{1} \mathrm{~d}^{2}+\beta_{2} \mathrm{~d}^{2} \mathrm{~h}+\beta_{3} \mathrm{~h}+\varepsilon_{\mathrm{i}}$ \\
7 & Meyer & $\mathrm{v}=\beta_{0}+\beta_{1} \mathrm{~d}+\beta_{2} \mathrm{~d}^{2}+\beta_{3} \mathrm{dh}+\beta_{4} \mathrm{~d}^{2} \mathrm{~h}+\beta_{5} \mathrm{~h}+\varepsilon_{\mathrm{i}}$ \\
8 & Schumacher - Hall & $\ln (\mathrm{v})=\beta_{0}+\beta_{1} \ln (\mathrm{d})+\beta_{2} \ln (\mathrm{h})+\varepsilon_{\mathrm{i}}$ \\
9 & Spurr logaritimica & $\ln (\mathrm{v})=\beta_{0}+\beta_{1} \ln \left(\mathrm{d}^{2} \mathrm{~h}\right)+\varepsilon_{\mathrm{i}}$ \\
10 & Prodan & $\ln (\mathrm{v})=\beta_{0}+\beta_{1} \ln (\mathrm{d})+\beta_{2} \ln \left(\mathrm{d}^{2}\right)+\beta_{3} \ln (\mathrm{h})+\beta_{4} \ln \left(\mathrm{h}^{2}\right)+\varepsilon_{\mathrm{i}}$ \\
\hline
\end{tabular}

* Em que: In = logaritmo neperiano; $v=$ volume $\left(\mathrm{m}^{3}\right) ; \mathrm{d}=$ diâmetro medido a $1,3 \mathrm{~m}$ do solo $(\mathrm{cm}) ; \mathrm{h}=$ altura do fuste $(\mathrm{m}) ; \beta_{0}, \beta_{1}, \beta_{2}, \beta_{3}$ e $\beta_{4}=$ parâmetros a serem estimados; $\varepsilon_{\mathrm{i}}=$ erro aleatório, $\varepsilon \mathrm{i} \sim \mathrm{NID}\left(0, \sigma^{2}\right)$.

forem não significativos (ns) e $r_{Y_{i} Y_{1}} \geq 1-|\bar{e}|$, conclui-se que os valores estimados pela regressăo não diferem dos valores observados.

\section{RESULTADOS E DISCUSSÃO}

Os fatores de forma médios calculados para árvores individuais com e sem casca foram de 0,7424 e 0,7297, respectivamente.

As árvores amostradas apresentaram valores médios de 66,45 e 63,92 cm de dap com e sem casca, respectivamente, $16,3 \mathrm{~m}$ de altura total média. O volume real médio com casca foi de $5,76 \mathrm{~m}^{3}$, com erro padrão da média igual a $0,069 \mathrm{~m}^{3}$. O volume de casca médio foi de $0,4292 \mathrm{~m}^{3}$ com desvio-padrão de $\pm 0,4696 \mathrm{~m}^{3}$.
O valor da constante de casca (k) foi de 0,9620 , dessa forma a porcentagem de casca para o conjunto de árvores cubadas foi de $7,45 \%$.

Após o ajuste das equações procedeu-se a análise dos parâmetros dos modelos, e selecionando-se aqueles que apresentaram melhores desempenhos na estimativa do volume para árvores individuais.

O coeficiente de determinação ajustado, o erro padrão da estimativa, bem como os coeficientes da regressão para os modelos volumétricos com e sem casca são apresentados nas Tabelas 4 e 5 , respectivamente.

Analisando as Tabelas 4 e 5 e as Figuras 1 e 2, de maneira geral os modelos testados apresentaram ajustes aos dados superiores a $90 \%$. Contudo ao avaliar o comportamento das distribuições dos resíduos selecionaram-se, preliminarmente,

Tabela 4 - Coeficientes estimados e medidas de precisão (coeficiente de determinação ajustado $-\mathrm{R}_{\text {ajustado }}^{2}$ e erro padrão de estimativa $-\mathrm{S}_{\mathrm{yx}}$ ) para os modelos volumétricos com casca*

\begin{tabular}{|c|c|c|c|c|c|c|c|c|}
\hline \multirow{2}{*}{ Modelo } & \multicolumn{6}{|c|}{ Coeficientes estimados } & \multirow{2}{*}{$\begin{array}{c}\mathrm{R}_{\text {ajustado }}^{2} \\
(\%)\end{array}$} & \multirow{2}{*}{$\begin{array}{c}S_{x y} \\
\left(m^{3}\right)\end{array}$} \\
\hline & $\beta_{0}$ & $\beta_{1}$ & $\beta_{2}$ & $\beta_{3}$ & $\beta_{4}$ & $\beta_{5}$ & & \\
\hline 1 & $-0,4306$ & 0,0011 & & & & & 92,8 & $\pm 1,68$ \\
\hline 2 & $-0,1076$ & $-0,0098$ & 0,0012 & & & & 92,7 & $\pm 1,69$ \\
\hline 3 & $-7,9906$ & 2,2416 & & & & & 92,4 & $\pm 1,72$ \\
\hline 4 & $-8,207$ & 2,2853 & 1,8864 & & & & 92,2 & $\pm 1,75$ \\
\hline 5 & 0,4413 & 0,0001 & & & & & 95,9 & $\pm 1,26$ \\
\hline 6 & $-1,2407$ & 0,0005 & $2,88.10^{-5}$ & 0,0887 & & & 96,9 & $\pm 1,10$ \\
\hline 7 & 2,1163 & $-0,1089$ & 0,0012 & 0,0061 & $-0,0001$ & $-0,1183$ & 97,0 & $\pm 1,08$ \\
\hline 8 & $-9,1892$ & 1,9693 & 0,837 & & & & 96,6 & $\pm 1,16$ \\
\hline 9 & $-9,3381$ & 0,9609 & & & & & 96,1 & $\pm 1,24$ \\
\hline 10 & $-9,1830$ & 0,6096 & 0,6817 & 0,2861 & 0,2680 & & 95,7 & $\pm 1,15$ \\
\hline
\end{tabular}

* Em que: 1) Kopezky - Gehrhardt; 2) Hohenald - Krenn; 3) Husch; 4) Brenac; 5) Spurr; 6) Stoate; 7) Meyer; 8) Schumacher - Hall; 9) Spurr logaritimica; 10) Prodan. 
Tabela 5 - Coeficientes estimados e medidas de precisão (coeficiente de determinação ajustado $-R_{\text {aiustado }}^{2}$ e erro padrão de estimativa $-S_{y x}$ ) para os modelos volumétricos sem casca*.

\begin{tabular}{|c|c|c|c|c|c|c|c|c|}
\hline \multirow{2}{*}{ Modelo } & \multicolumn{6}{|c|}{ Coeficientes estimados } & \multirow{2}{*}{$\begin{array}{c}\mathrm{R}_{\text {aiustado }}^{2} \\
(\%)\end{array}$} & \multirow{2}{*}{$\begin{array}{l}S_{x y} \\
\left(m^{3}\right)\end{array}$} \\
\hline & $\beta_{0}$ & $\beta_{1}$ & $\beta_{2}$ & $\beta_{3}$ & $\beta_{4}$ & $\beta_{5}$ & & \\
\hline 1 & $-0,1796$ & 0,001 & & & & & 91,2 & $\pm 1,64$ \\
\hline 2 & $-0,5977$ & 0,0133 & 0,001 & & & & 91,2 & $\pm 1,64$ \\
\hline 3 & $-7,9805$ & 2,237 & & & & & 90,0 & $\pm 1,76$ \\
\hline 4 & $-7,9214$ & 2,225 & $-0,4941$ & & & & 90,1 & $\pm 1,75$ \\
\hline 5 & 0,5426 & $4,82 \cdot 10^{-5}$ & & & & & 95,5 & $\pm 1,18$ \\
\hline 6 & $-1,3807$ & 0,0004 & $2,74.10^{-5}$ & 0,1133 & & & 96,3 & $\pm 1,07$ \\
\hline 7 & 1,8333 & $-0,0682$ & 0,0073 & 0,0059 & $-1,04.10^{-6}$ & $-0,162$ & 96,5 & $\pm 1,04$ \\
\hline 8 & $-9,198$ & 1,9734 & 0,8268 & & & & 95,7 & $\pm 1,16$ \\
\hline 9 & $-9,3663$ & 0,9615 & & & & & 95,4 & $\pm 1,19$ \\
\hline 10 & $-9,1821$ & 0,6465 & 0,6613 & 0,3041 & 0,2562 & & 96,6 & $\pm 1,16$ \\
\hline
\end{tabular}

* Em que: 1) Kopezky - Gehrhardt; 2) Hohenald - Krenn; 3) Husch; 4) Brenac; 5) Spurr; 6) Stoate; 7) Meyer; 8) Schumacher - Hall; 9) Spurr logaritimica; 10) Prodan.

1

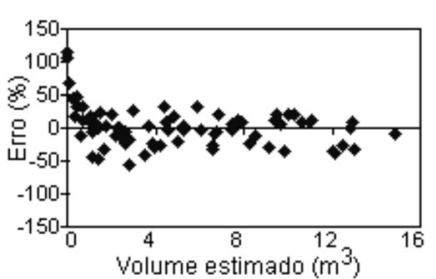

4

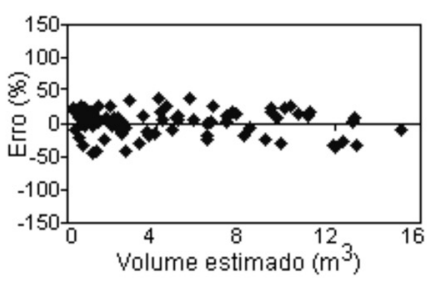

7
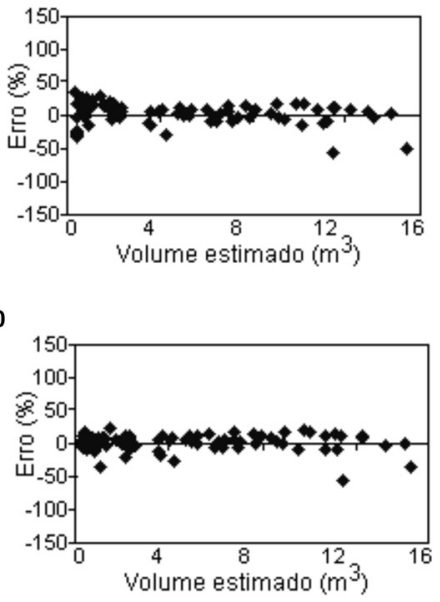
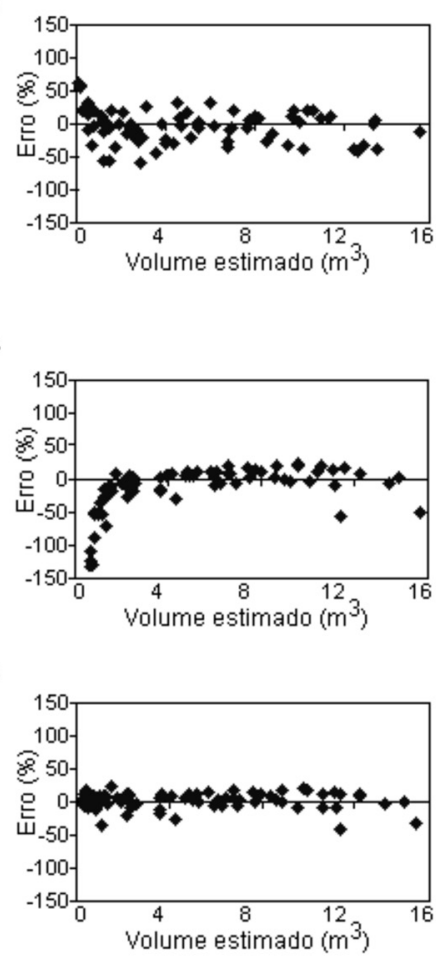

3

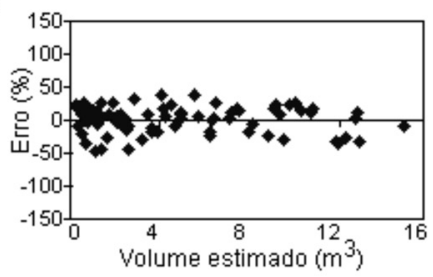

6

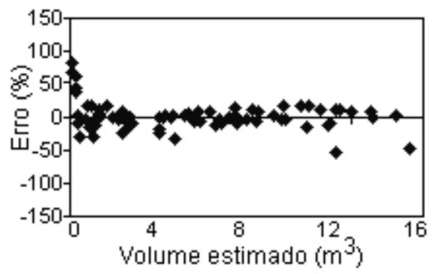

9

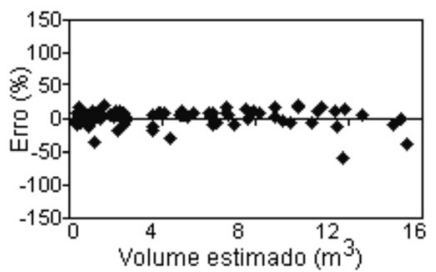

Figura 1 - Resíduos em função do volume estimado para os modelos volumétricos com casca. Em que: 1) Kopezky - Gehrhardt; 2) Hohenald - Krenn; 3) Husch; 4) Brenac; 5) Spurr; 6) Stoate; 7) Meyer; 8) Schumacher - Hall; 9) Spurr logaritimica; 10) Prodan. 

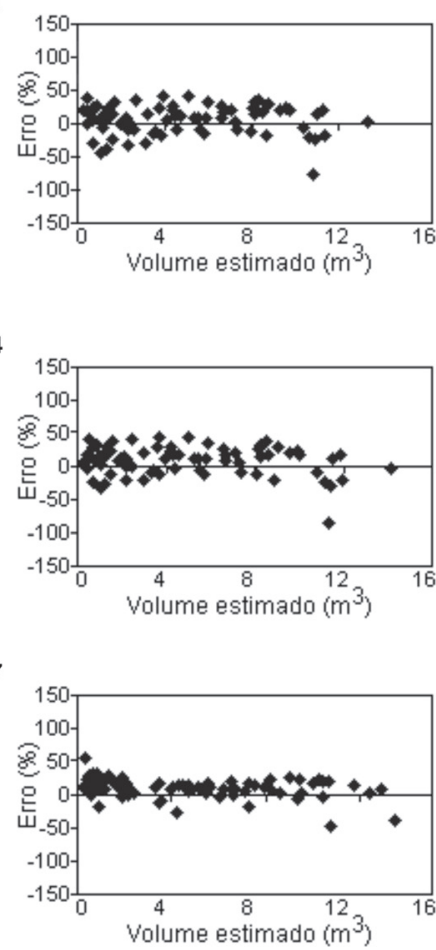

10

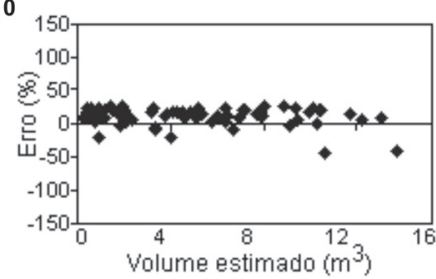

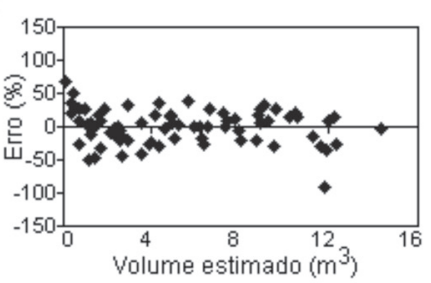

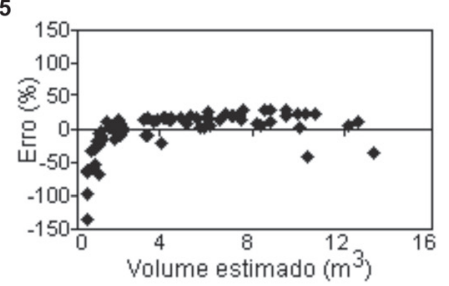

8

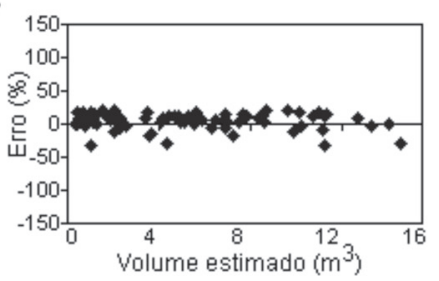

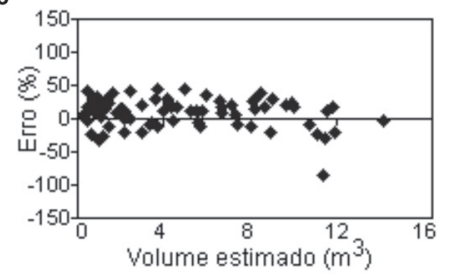

6

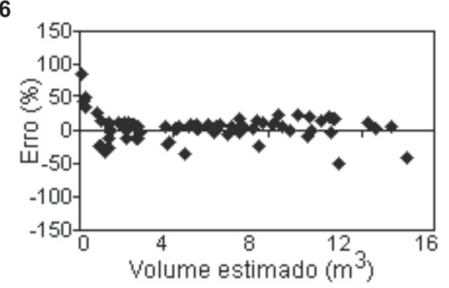

9

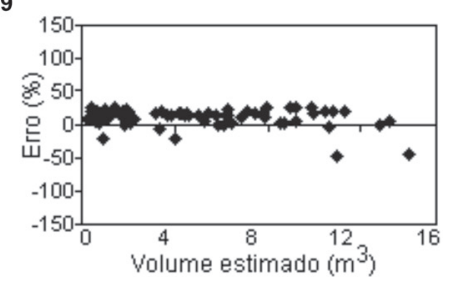

Figura 2 - Resíduos em função do volume estimado para os modelos volumétricos sem casca. Em que: 1) Kopezky - Gehrhardt; 2) Hohenad - Krenn; 3) Husch; 4) Brenac; 5) Spurr; 6) Stoate; 7) Meyer; 8) Schumacher - Hall; 9) Spurr logaritimica; 10) Prodan.

as equações 8 (Schumacher-Hall) e 10 (Prodan) tanto para os volumes com e sem casca, pois apresentaram os melhores ajustes. A equação 8 apresenta o melhor comportamento residual, pois está mais bem distribuído ao longo do eixo e sem apresentar grandes desvios em torno do eixo zero já o modelo 10 possui uma tendência a subestimar os volumes quando comparado ao modelo 8 .
Observando os resultados do teste de L\&O (Tabela 6), verifica-se que as equações obtidas a partir do modelo de Schumacher-Hall apresentaram estimativas precisas na predição dos volumes com e sem casca. Por outro lado, o volume sem casca estimado pela equação de Prodan foi estatisticamente diferente do volume observado.

Analisando as medidas de precisão das equações selecionadas e o resultado do teste de $\mathrm{L} \& \mathrm{O}$, verifica-se que a

Tabela 6 - Estatísticas do teste de L\&0 para validação das equações ajustadas, considerando o volume observado e 0 volume estimado, para um nível de significância de $5 \%$.

\begin{tabular}{|c|c|c|c|c|c|}
\hline Situação & Equação & $\mathrm{F}\left(\mathrm{H}_{0}\right)$ & $\bar{e}$ & $r_{Y_{j} Y_{1}} \leq(1-|\bar{e}|)$ & Conclusão* \\
\hline \multirow{2}{*}{ Com casca } & Schumacher-Hall & $0,018 n s$ & $0,121 \mathrm{~ns}$ & $\operatorname{sim}$ & $Y_{j}^{*}$ é estatisticamente igual a $Y_{1}^{* *}$, para o nível de significância estabelecido. \\
\hline & Prodan & $0,018 \mathrm{~ns}$ & $0,130 \mathrm{~ns}$ & $\operatorname{sim}$ & $Y_{j}^{*}$ é estatisticamente igual a $Y_{1}^{* *}$, para o nível de significância estabelecido. \\
\hline \multirow{2}{*}{ Sem casca } & Schumacher-Hall & $0,019 n s$ & $1,226 n s$ & $\operatorname{sim}$ & $Y_{j}^{*}$ é estatisticamente igual a $Y_{1}^{* *}$, para o nível de significância estabelecido. \\
\hline & Prodan & $0,019 n s$ & $6,158^{*}$ & $\operatorname{sim}$ & $Y_{j}^{*}$ é estatisticamente diferente $a Y_{1}^{* *}$, para o nível de significância estabelecido. \\
\hline
\end{tabular}

$\star Y^{*}=$ valores observados

$\star \star Y_{1}^{j}=$ valores estimados 
equação de Schumacher-Hall é a que permite obter estimativas mais precisas dos volumes, com e sem casca, individuais na área em estudo, por não apresentar tendências nítidas de superestimar ou subestimar os volumes dentro da amplitude de diâmetros observados.

\section{CONCLUSÕES}

De acordo com os resultados apresentados no presente estudo, pode-se concluir que:

- A equação de Schumacher-Hall apresenta maior precisão para estimar o volume em função do diâmetro e da altura total das árvores, sendo a mesma selecionada e, por isso, recomendada para a área estudada.

- A percentagem média de casca em espécies da floresta estudada foi relativamente baixa, porém em conformidade com inúmeras outras pesquisas em florestas tropicais.

- O fator de forma médio encontrado nesta pesquisa se aproxima bastante dos encontrados por Heinsdijk (1962) e por Moura (1994) para espécies da floresta amazônica

\section{BIBLIOGRAFIA CITADA}

APG II. 2003. An update of the Angiosperm Phylogeny Group classification for the orders and families of flowering plants: APG II. Botanical Journal of the Linnean Society, 141 (4): 399-436.

Bruce, D.; Schumacher, F.X. 1950. Forest mensuration. McGraw-Hill, New York. 483p.

Chapman, H.H.; Meyer, W.H. 1949. Forest mensuration. McGrawHill, New York. 522p.

Draper, N.; Smith, H. 1998. Applied regression analysis. John Wiley \& Sons, New York. 736p.

Heinsdijk, D. 1963. Inventários florestais na Amazônia. Ministério da Agricultura, Serviço Florestal Brasileiro, Rio de Janeiro. 100p. (Boletim, 6)

Higuchi, N., Gomes, B.; Santos, J.; Constantino, N.A. 1979. Tabela de volume para povoamento de Eucalyptus grandis plantado no município de Várzea Grande (MT). Floresta, 10(1): 43-47.

Leite, H.G.; Oliveira, F.H.T. 2002. Statistical procedure to test the identity of analytical methods. Communications in Soil Science and Plant Analysis, 7/8: 1 - 23.

Machado, S.A. 1979. Estimativa de sobrevivência de Pinus taeda em plantios homogêneos. Floresta, 10(1): p.73-76.
Machado, S.A.; Figueiredo Filho, A. 2003. Dendrometria. [s.e.], Curitiba. 309pp.

Machado, S.A.; Urbano, E; Conceição, M.B.; Figueiredo Filho, A.; Figueiredo, D.J. 2004. Comparação de modelos de afilamento do tronco para diferentes idades e regimes de desbaste em plantações de Pinus oocarpa Schiede. Boletim de Pesquisa Florestal, 48: 41-64.

Moura, J.B. 1994. Estudo de forma do fuste e comparação de métodos de estimativa volumétrica de espécies florestais da Amazônia Brasileira. Dissertação de Mestrado, Universidade Federal do Paraná, Curitiba, Paraná, 114pp.

Paula Neto, F. 1977. Tabelas volumétricas com e sem casca para Eucalyptus saligna. Árvore, 1(1): 31-54.

Prodan, M. 1968. Forest biometrics. Trad. Sabine H. Gardiner Pergamon Press, Oxford. 447p.

Scolforo, J.R.S. 1997. Manejo florestal. UFLA/FAEPE, Lavras. $443 \mathrm{p}$.

Scolforo, J.R.S.; Mello, J.M.; Lima, C.S.A. 1994. Obtenção de relaçóes quantitativas para estimativa de volume do fuste em floresta estacional semidecídua montana. Cerne, 1(1): 123134.

Scolforo, J.R.S.; Thiersch, C.R. 2004. Biometria florestal: medição, volumetria e gravimetria. UFLA/FAEPE, Lavras. 285p.

Siqueira, J.P.D. 1977. Tabelas de volume para povoamentos nativos de Araucária angustifolia (Bert) O, Ktze, no sul do Brasil. Dissertação de Mestrado, Universidade Federal do Paraná, Curitiba, Paraná, 163pp.

Spurr, S.H. 1952. Forestry inventory. Ronald Press, New York. 476p.

Thomas, C.; Andrade, C.M.; Schneider, P.R.; Finger, C.A.G. 2006. Comparação de equações volumétricas ajustadas com dados de cubagem e análise de tronco. Ciência Florestal, 16(3): 319-327.

Recebido em 23/04/2008

Aceito em 26/06/2008 